\title{
Afeto como um Valor no Design de Tecnologias Educacionais: frameworks e discussões informadas
}

\author{
Roberto Pereira, Elaine C. S. Hayashi, M. Cecília C. Baranauskas \\ Instituto de Computação, UNICAMP \\ \{rpereira, hayashi, cecilia\}@ic.unicamp.br
}

\begin{abstract}
The research communities in HCI and Informatics in Education have been showing a crescent interest in the investigation and discussion of social and affective aspects in educational technology. In this article we promote a discussion where affect is taken as a value and, for that, we articulate two conceptual frameworks: Value Pie and the Framework for the Design for Affectibility, which support and inform the design focusing on values and affect, respectively. This discussion innovates in the articulation of these two frameworks and it contributes with theoretical and practical suggestions for designers. Moreover, it provides base to construct a new perspective in the way we handle affective issues in the design of computational systems.
\end{abstract}

Resumo. As comunidades de pesquisa em IHC e Informática na Educação vêm mostrando crescente interesse em investigar e discutir aspectos sociais e afetivos em tecnologias educacionais. Entendendo Afeto como Valor, promovemos neste artigo uma discussão que articula as contribuições de dois frameworks conceituais: o Value Pie e o Framework para Design para Afetibilidade - frameworks que apoiam e informam o design com foco em valores e afeto, respectivamente. Esta discussão inova ao articular esses dois frameworks e contribui ao oferecer sugestões de caráter teórico e prático para designers, além de servir como base para uma nova perspectiva na forma de tratar questões afetivas no design de sistemas computacionais.

\section{Introdução}

O poder que o uso de tecnologias digitais exerce na vida das pessoas tem importância reconhecida, afetando diretamente a sociedade que as adota [Friedman et al., 2006; Boehner et al., 2007]. Neste processo, as escolhas de design presentes nas tecnologias podem ser determinantes. Ao favorecer ou inibir valores de relevância para aquela sociedade, as escolhas de design presentes nas tecnologias não apenas refletem a sociedade que as criou, como também passam a moldá-la.

Hall afirma que "a educação e os sistemas educacionais são tão carregados de emoção e tão característicos de uma cultura como sua própria linguagem" (Hall, 1959, p.47). Deste modo, nós argumentamos que, no design de tecnologias educacionais, é preciso discutir e lidar com essas questões sob a perspectiva de valores culturais, de forma transversal a todas as etapas do ciclo de design, e se preocupando com a maneira como os participantes são envolvidos e tratados nesse ciclo.

Um dos grandes desafios ao lidar com aspectos emocionais e afetivos, é que problemas relacionados a esses aspectos são normalmente muito sutis para serem notados até que algum conflito efetivamente ocorra. Isso se deve, em partes, porque as discussões e análises sobre emoção e afeto em tecnologia tendem a ocorrer após a 
concepção e lançamento de um produto. No entanto, embora a negligência com essas questões seja preocupante em qualquer contexto, ela é particularmente prejudicial no projeto de tecnologias educacionais, tanto por impactarem diretamente em crianças quanto por influenciarem as práticas de ensino e aprendizagem de um grupo social.

Embora reconhecidos como importantes, fatores emocionais e afetivos, valores e cultura são aspectos tradicionalmente deixados às margens dos processos de construção de sistemas interativos em geral. Buchdid e Baranauskas (2012) mostram indícios de que esses aspectos não têm aparecido de forma significativa na literatura recente.

Neste artigo, apresentamos uma discussão sobre afeto do ponto de vista de valores em tecnologias educacionais. Nossa discussão é fundamentada por dois diferentes frameworks, teórica e metodologicamente informados, que apoiam o design sob a perspectiva de afeto e de valores: o Value Pie (VP) e o Design para Afetibilidade (FDAf). O VP e o FDAf buscam trazer e envolver elementos importantes de diferentes partes interessadas (e.g., afeto, valores, cultura) no processo de design de tecnologias. Enquanto o primeiro favorece a discussão da Afetibilidade como um valor em sistemas interativos (o que deve ser considerado e porque deve ser considerado), o segundo favorece o envolvimento dessas questões nas diferentes etapas de design (da clarificação do contexto do problema até a avaliação do sistema projetado). Como contribuição deste artigo, apresentamos e articulamos estes frameworks, e sugerimos pontos importantes a serem considerados em atividades de design com foco em questões afetivas. Até onde estamos cientes, não há outros trabalhos na literatura atual que articulem afeto, valores e cultura de forma explícita e informada.

Este artigo está organizado da seguinte forma: na seção 2 apresentamos a fundamentação teórica do trabalho, descrevendo os dois frameworks utilizados. Na seção 3, discutimos afeto e Afetibilidade como valores no design de tecnologias educacionais, chamando atenção para os valores associados que devem ser considerados pelos designers. Na seção 4, discutimos como esses valores podem ser envolvidos dentro do ciclo de design para Afetibilidade. Finalmente, na seção 5, apresentamos as conclusões deste trabalho.

\section{Fundamentação teórica}

Valor é tudo aquilo que uma sociedade considera importante [Friedman et al., 2006]. Segundo Schwartz (2005), valores são crenças particulares de cada cultura e comunidade, motivam ações, transcendem especificidades de determinadas situações e servem de critérios-base para formação de julgamentos. Desta forma, valores são cruciais para explicar as organizações sociais e sua evolução.

A compreensão do funcionamento das organizações é essencial no design de sistemas computacionais que façam sentido para essas organizações. Podemos entender como organizações não apenas empresas, mas também comunidades, como, por exemplo, as comunidades escolares [Hayashi et al., 2012]. Identificar e entender quais são os valores de maior relevância no contexto de uma organização é um desafio que o VP [Pereira et al., 2013] visa apoiar. Sem o intuito de ser exaustivo, o framework (Figura 1) permite a discussão de valores de acordo com 3 dimensões: 1. Cultura valores são desenvolvidos de acordo com diferentes "áreas" que caracterizam uma cultura: interação, associação, aprendizado, diversão, proteção, exploração, temporalidade, territorialidade, subsistência, classificação; essas áreas são representadas 
por "fatias" no VP. 2. Formalidade - valores se manifestam e devem ser entendidos em 3 diferentes níveis: informal, formal e técnico; esses níveis são representados por camadas no VP. 3. Inter-relacionamento - valores são ligados uns aos outros de acordo com sua natureza cultural (e.g., valores desenvolvidos em uma mesma área).

A Figura 1 apresenta, organizados de acordo com o VP (Pereira et al., 2013), um conjunto de valores identificados no contexto de software social. Relacionados com o nível informal estão os valores de natureza pessoal; com o nível formal, os valores ligados a normas sociais ou sistemas de leis; e com o nível técnico, estão os valores que podem ser entendidos como características ou atributos de qualidade de uma aplicação tecnológica. Os valores situados em uma mesma área de cultura possuem a mesma natureza ou raiz cultural. Por exemplo, os valores de privacidade, consentimento informado e segurança estão ligados ao conceito cultural de salvaguarda, proteção. Em um nível informal, cada indivíduo ou comunidade tem sua concepção do que é correto quanto ao tratamento, direitos e deveres das crianças. No nível formal, temos a Declaração dos Direitos da Criança (ONU), que enumera alguns desses direitos e deveres, segundo um consenso da comunidade internacional. No nível técnico, podemos mencionar como exemplo os Termos de Uso de aplicações ou páginas da internet que regulam ou alertam sobre a participação ou exposição de crianças. Finalmente, os valores em uma mesma área possuem um relacionamento cultural entre si (e.g., grupos são formados por relacionamentos comuns entre os indivíduos; a conversação entre indivíduos de um mesmo grupo pode promover a confiança entre eles, etc.).

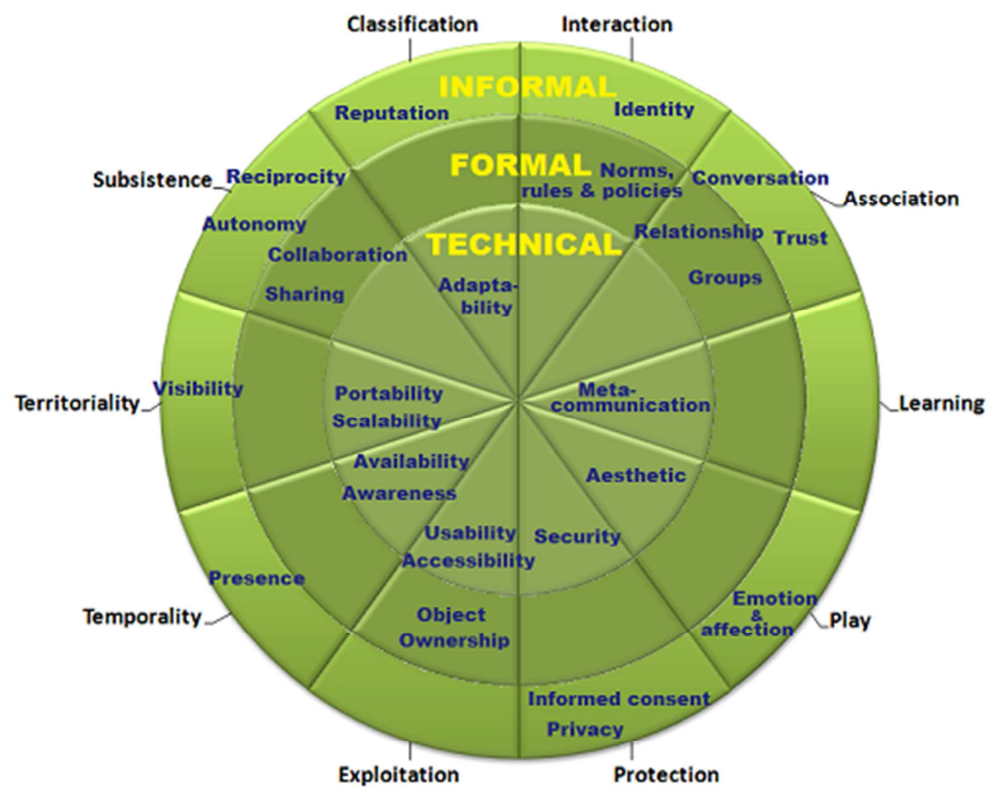

Figura 1. Value Pie

A principal contribuição do VP está em concientizar designers sobre os valores a serem considerados no design da tecnologia e sua natureza cultural. Entre os valores indicados no VP estão emoção e afeto. Neste artigo, adotamos a definição de afeto proposta por Ortony et al. (2005), que considera afeto como um construto geral que inclui uma ampla gama de estados psicológicos relacionados a valor, tais como emoções, sentimentos, temperamentos, preferências, etc.

Ao pensarmos em afeto como um valor a ser considerado no contexto do design 
de tecnologias educacionais, podemos notar que afeto está ligado a valores de outras fatias e camadas. $\mathrm{O}$ afeto está diretamente ligado às complexidades do contexto cultural inerente aos processos de design para tecnologias do ambiente escolar. Para compreender como afeto e demais valores relacionados influenciam e são influenciados pela comunidade escolar e suas tecnologias é preciso buscar conhecer as diferentes partes interessadas, suas particularidades culturais, expectativas, necessidades, limitações, enfim, todo o ecossistema no qual a comunidade está imersa. Atingir tal conhecimento e aplicá-lo no design de interação é vital para a produção de sistemas interativos que sejam não apenas operáveis, mas que, principalmente, façam sentido para os usuários, atendendo suas expectativas e favorecendo o processo de apropriação. Como fazer isso é um desafio que o FDAf [Hayashi e Baranauskas, 2013] visa apoiar.

O Design para Afetibilidade pode ser entendido como os processos de design de interação preocupados com as respostas afetivas dos usuários finais da tecnologia (e.g., alunos, professores, outros profissionais da educação e outros membros da comunidade escolar). A resposta afetiva das partes interessadas é importante tanto no uso da aplicação final quanto durante os processos de criação, durante as práticas de design participativo, de outros métodos de pesquisa ou de design que os envolvam.

Assim como o VP, o FDAf também considera os 3 níveis de formalidade (informal, formal e técnico) e sugere etapas, dentro do ciclo do Design Socialmente Responsável proposto por Baranauskas (2009), que trazem as respostas afetivas para dentro do processo de design. A Figura 2 ilustra esse processo.

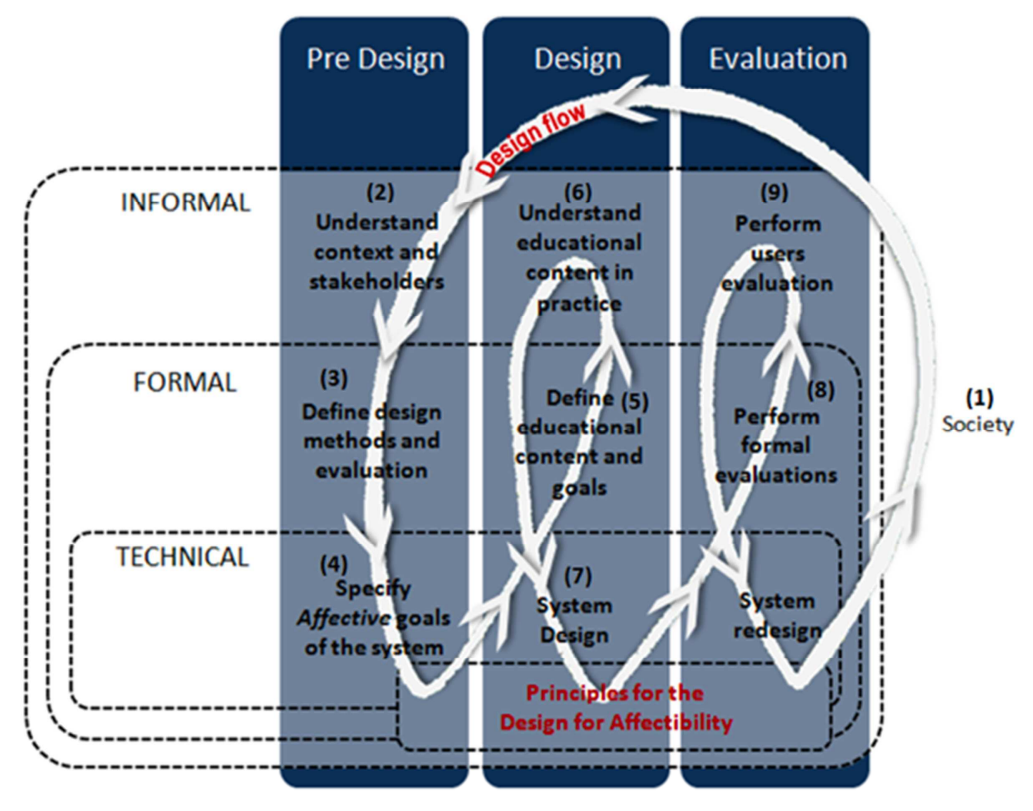

Figura 2 - Framework para o Design para Afetibilidade [Hayashi e Baranauskas, 2013].

O ciclo do Design Socialmente Responsável, representado no FDAf, sugere que o processo de design tem início na sociedade, passa pelas camadas informal e formal em direção à criação de uma solução tecnológica na camada técnica. $\mathrm{O}$ ciclo continua, retornando pelas camadas formal e informal e impactando a sociedade na qual o ciclo foi iniciado. O FDAf indica os passos do processo de design para Afetibilidade dentro desse ciclo, organizando-os em três colunas: pré-design, design e avaliação. 
O início do processo de design é marcado pelas oportunidades ou necessidades/problemas que dão razão para a criação de um sistema computacional. Elas nascem das especificidades - habilidades, carências, desejos de uma sociedade, ver detalhe (1) na Figura 2. Para aproveitar tais oportunidades, suprir necessidades, ou resolver problemas de maneira eficaz, é preciso compreender o contexto (2) de uso e as partes envolvidas, estejam elas direta ou indiretamente relacionadas ao problema ou sua solução. O caminho para se obter essa compreensão deve ser escolhido dentre os métodos de pesquisa, de design e de avaliação mais apropriados para cada situação ou interesse (3). A partir do conhecimento construído nos passos anteriores é possível especificar os objetivos afetivos do sistema (4). Nesse passo, o designer deve questionar e refletir sobre o tipo de resposta afetiva que se espera favorecer durante interação dos usuários com o sistema. Ao mesmo tempo, é preciso definir o conteúdo e os objetivos educacionais do sistema (5), que devem ser confirmados na prática escolar (6). Uma vez definidos, os objetivos afetivos e educacionais devem ser materializados de algum modo nos recursos de interface/interação do sistema sendo projetado (7). Os passos referentes à avaliação formal (8) e avaliação com usuários (9), cujos métodos foram escolhidos no passo (3), também têm a sua base no conceito de Afetibilidade.

Desta forma, deve-se dedicar uma atenção cuidadosa às respostas afetivas das pessoas envolvidas na tarefa de avaliação, sejam usuários finais do sistema ou especialistas. O produto resultante do redesign do sistema feito com base nos resultados das avaliações volta para a sociedade que, ao fazer uso do sistema e incorporá-lo em suas práticas, cria uma nova cultura em torno do artefato, podendo gerar novos comportamentos e hábitos, criando, por sua vez, novas habilidades e necessidades, dando continuidade ao ciclo.

\section{Valores e o design para Afetibilidade}

Considerar afeto como um valor contribui para um melhor entendimento de como efetivamente considerá-lo e envolvê-lo no processo de design de tecnologias educacionais. O VP indica que afeto é um valor relacionado a questões culturais de diversão (Play) e que se manifesta no nível informal, junto com as crenças, expectativas, hábitos e outros padrões de comportamento das pessoas. Valores, tais como identidade, confiança e relacionamento são claramente caracterizados por aspectos emocionais e afetivos; outros valores, como privacidade, reputação e reciprocidade são fortemente relacionados com questões afetivas, influenciando e sendo influenciado por elas.

O conceito de Afetibilidade pode ser entendido como a representação do valor afeto no nível técnico. Afetibilidade está relacionada às características de um artefato digital que ajudam a disparar respostas afetivas durante as interações que ocorrem no micro-ecossistema formado por usuários, sistema e comunidade escolar [Hayashi e Baranauskas, 2013]. Entre as respostas afetivas estão as respostas emocionais, hedônicas, estéticas, etc. Dependendo do momento e objetivos de uma atividade, diferentes respostas podem ser esperadas. Por exemplo, ansiedade pode ser a resposta almejada em certos momentos de decisão em um jogo, porém a ser evitada em outros momentos onde maior concentração e paciência são exigidos.

Considerando o VP, é possível observar que o valor Afetibilidade também pode influenciar e ser influenciado pelos valores Usabilidade e Acessibilidade, apresentados na camada "Exploração". De um lado, a promoção desses valores tende a produzir uma 
resposta positiva no modo como as pessoas se sentem com relação ao sistema. De outro lado, se a usabilidade e a acessibilidade de um sistema interativo forem negligenciadas, então a resposta afetiva evocada pelo sistema tende a ser negativa, potencializando a rejeição ao sistema.

Outros valores apresentados no VP são facilmente relacionados com Afetibilidade. Por exemplo, o valor Adaptabilidade, apresentado na área "Classificação", pode favorecer a Afetibilidade de um sistema interativo ao oferecer recursos de personalização da interface, ao evitar informação desnecessária, ao facilitar a interação do usuário, etc. O entendimento de Normas, regras sociais, códigos de conduta, etc. (Normas é um valor na camada "Interação"), também é essencial para o design de um sistema que promova o valor Afetibilidade. Caso contrário, corre-se o risco de se projetar um sistema que conflite com as normas de uma sociedade, desencadeando impactos negativos. A Figura 3 apresenta o valor Afetibilidade juntamente com as áreas da cultura de Hall (1959), alguns valores do VP (Pereira et al., 2013) e outros termos relevantes para a Afetibilidade em cada uma das áreas.

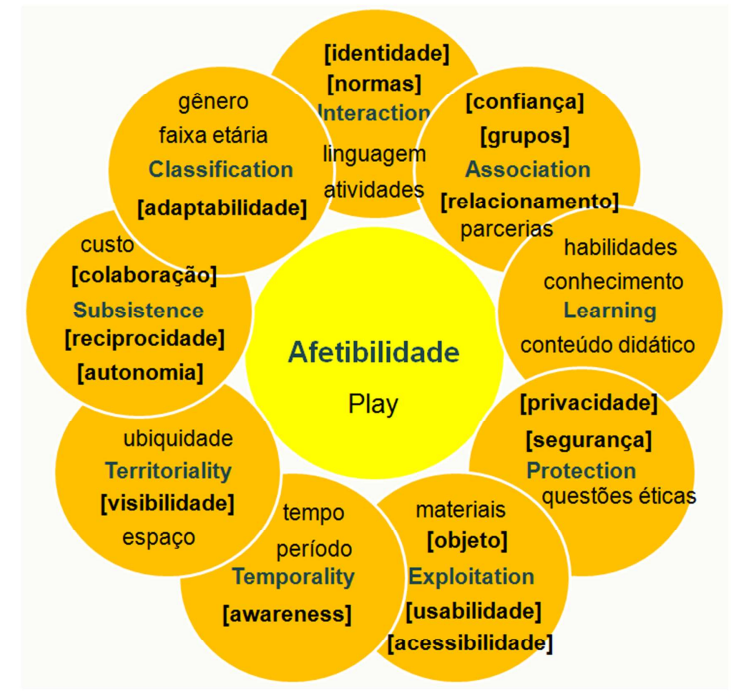

Figura 3. O valor Afetibilidade de acordo com as áreas da cultura e os valores do VP.

$\mathrm{Na}$ Figura 3 apresentamos o valor Afetibilidade no centro das áreas da cultura para demonstrar que, ao pensar em Afetibilidade, deve-se considerar a sua relação com a forma como as pessoas interagem, se organizam socialmente, aprendem, se protegem, exploram o mundo, entendem tempo e espaço, sobrevivem e colaboram, e classificam coisas e pessoas ao seu redor. Em cada área são apresentados valores vindos do VP (em negrito, entre colchetes) e outros termos relevantes relacionados à Afetibilidade.

Os termos e valores evidenciam a complexidade de questões que sofrem e/ou exercem influência sobre a Afetibilidade de uma tecnologia educacional. Por exemplo, além dos valores já mencionados anteriormente, a área "Classificação" indica a importância de se considerar diferenças de gênero e de faixa etária ao se pensar na Afetibilidade que uma tecnologia educacional pretende promover. Meninos e meninas, de diferentes faixas etárias, reagirão de forma diferente não apenas ao conteúdo apresentado e às atividades propostas, mas à própria interface apresentada pela tecnologia educacional. A área "Aprendizagem" sugere que, além do conteúdo didático, é preciso considerar o conhecimento prévio dos alunos, as habilidades cognitivas, as 
limitações, entre outros. Finalmente, a área "Proteção" chama a atenção para questões éticas envolvidas em todas as etapas do design de uma tecnologia educacional, incluindo as intenções existentes antes do início do processo, e os impactos que podem ser desencadeados após seu término e utilização.

\section{Considerações sobre Afetibilidade no processo de design}

Alcançar a Afetibilidade necessária e desejada em uma tecnologia educacional requer, também, um processo de design bem definido. O FDAf (ver Figura 2) sugere 9 etapas em um ciclo iterativo e incremental de design que contribuem para o entendimento do contexto social das partes interessadas envolvidas, de seus aspectos afetivos, valores, entre outras características culturais, apoiando os designers em suas atividades práticas.

No design de tecnologias educacionais, mais especificamente em contextos que envolvam crianças, a importância e o desafio de pensar em afeto são ainda maiores. $\mathrm{O}$ desenvolvimento cognitivo das crianças está diretamente ligado ao seu desenvolvimento afetivo [LaTaille, 1992], porém a maior parte dos designers de tecnologia educacional dedica-se apenas ao conteúdo didático das aplicações, deixando afeto de lado.

Conforme argumentamos anteriormente, o início e o fim do processo de design de tecnologia está na sociedade. Os valores diretamente relacionados a esse $1^{\circ}$ passo do FDAf são, em sua maioria, valores da camada informal do VP — sendo os relacionados à Proteção um dos mais críticos. Nesse sentido, durante todo o processo de design é preciso atentar para o bem estar dos participantes, garantindo seus direitos e atentando para os princípios éticos básicos (e.g. consentimento informado, possibilidade de desistência, liberdade de expressão e comunicação [Thomas e O'Kane, 1998]) no design para, com e por crianças.

A comunicação aberta facilita o $2^{\circ}$ passo do FDAf: compreender o contexto e as partes interessadas. Conhecer as expectativas, necessidades, limitações, além de saber o que é considerado importante para as diferentes partes interessadas favorece o design de soluções que façam sentido para elas. Valores relacionados a este passo estão, principalmente, entre as áreas "Associação" e "Interação" da Figura 3. Um artefato que pode auxiliar os designers é o Diagrama de Partes Interessadas [Liu, 2001], que organiza grupos de interessados de acordo com diferentes categorias favorecendo uma visão mais ampla do escopo do problema em questão. Outros artefatos, como o Quadro de Avaliação [Baranauskas et al., 2005] e o Quadro de Identificação de Valores [Pereira et al., 2012b] também podem auxiliar neste passo.

Com relação ao $3^{\circ}$ passo do FDAf, no qual os designers devem escolher métodos de design, Guha et al. (2010) sugerem que, para as atividades de design envolvendo a participação ativa de crianças, métodos qualitativos são mais adequados que métodos quantitativos, pois permitem que pesquisadores/designers estejam mais próximos do público-alvo. Exemplos: métodos etnográficos e entrevistas. Oficinas Semioparticipativas de design têm se mostrado como um modo eficiente de se fazer design socialmente responsável de forma participativa [Baranauskas et al., 2013; Melo et al., 2008].

No $4^{\circ}$ passo, a especificação dos objetivos afetivos do sistema é viabilizada, em partes, pelo conhecimento e proximidade das partes interessadas. Os Princípios de Design para Afetibilidade [Hayashi e Baranauskas, 2013] são um apoio adicional para os designers envolvidos nessa tarefa. Relacionados a este passo estão os valores de 
bem-estar, felicidade, motivação, envolvimento, controle, estética, etc. Para definir como o sistema poderá refletir os valores mais adequados à solução, o designer pode fazer perguntas como: o que traz bem-estar para os usuários? Com o que eles mais se empolgam ou se divertem? Quais suas preferências? Do que eles gostam e do que eles não gostam? O que os fazem sentir confortável em relação à interação com o sistema?

Com relação ao $5^{\circ}$ passo, os conteúdos e objetivos educacionais muitas vezes já vêm especificados como principais requisitos do sistema. Outras vezes, podem ser definidos com base no conhecimento que se tem das necessidades dos alunos, professores ou escolas, que foi clarificado nos passos anteriores. Em qualquer caso, é importante que o designer saiba como esses conteúdos são aplicados na prática $\left(6^{\circ}\right.$ passo). Isso permite o entendimento das normas informais que definem as interações do dia-a-dia nas salas de aula. Aqui, os elementos apresentados na área "Aprendizagem" da Figura 3 destacam quais valores devem ser considerados nas atividades de design.

No $7^{\circ}$ passo, referente à construção da solução, os objetivos afetivos do sistema devem ser representados por meio de recursos de interface e de interação de modo a refletir, direta e/ou indiretamente, esses objetivos. Além dos dados obtidos nos passos anteriores, o designer pode se apoiar nos Princípios para o Design para Afetibilidade [Hayashi e Baranauskas, 2013] para guiar seu trabalho. Diferentes técnicas e ferramentas podem ser utilizadas para apoiar este passo. Por exemplo, o Brain Draw é uma técnica participativa que favorece a criação colaborativa de protótipos de interface [Muller et al., 1997]; o CoTool $^{\circledR}$ é uma ferramenta que possibilita a criação de protótipos e a modelagem de tarefas, etc.

Para os passos 8 e 9, referentes a avaliação formal e informal, respectivamente, o designer pode se utilizar de diferentes técnicas e formas de avaliação. Avaliações formais podem ser conduzidas por meio de técnicas como as Heuristicas de Nielsen [Nielsen, 1994] e análise GOMS [John e Kieras, 1996]. O artefato eValue [Pereira et al., 2012a], construído sobre as bases teóricas e metodológicas do VP, pode ser utilizado para apoiar a avaliação da solução projetada sob a ótica de cultura e valores.

Avaliações informais podem ser conduzidas por meio de workshops com representantes do público-alvo, questionários online, entrevistas, etc. Nestes passos, assim como no redesign do sistema e em todo o ciclo de design representado na Figura 2, os designers também devem estar atentos aos valores em design e aos Princípios para Afetibilidade. O Self-Assessment Manikin [Bradley e Lang, 1994], Sensual Evaluation Instrument [Isbister et al., 2006] e o Affect Grid [Russell, 1989] são exemplos de artefatos que podem ser utilizados para entender ou coletar respostas afetivas.

\section{Conclusão}

É preciso entender e reconhecer que aspectos afetivos influenciam diretamente na aceitação de um sistema interativo e na forma como esse sistema é percebido, entendido e utilizado. A falta de atenção com esses aspectos tem levado à criação de produtos que não fazem sentido para seus usuários, que não atendem às expectativas de suas diferentes partes interessadas, e que, muitas vezes, desencadeiam impactos indesejados no ambiente em que são disponibilizados. Aspectos de afetividade dificilmente serão clarificados apenas por meio de uma entrevista com potenciais usuários ou de alguns estudos de campo; eles precisam ser trazidos à tona por meio de análises informadas e explicitamente preocupadas com valores e com sua natureza cultural. 
Neste artigo, articulamos dois frameworks conceituais, Value Pie e Design para Afetibilidade, que buscam trazer e envolver elementos importantes das diferentes partes interessadas (e.g., afeto, valores, cultura) no processo de design de tecnologias. Entendendo afeto como um valor, nos fundamentamos no VP para discutir a Afetibilidade em tecnologias educacionais e revisitamos os passos sugeridos pelo FDAf, citando artefatos e técnicas complementares.

A discussão de afeto do ponto de vista de valores e cultura é uma contribuição deste trabalho. Esperamos que as discussões apresentadas abram espaço para novos debates e novas formas de abordar a questão afetiva no design de tecnologias educacionais.

\section{Agradecimentos}

Este trabalho é apoiado parcialmente por CNPq (\#160819/2013-0) e FAPESP (\#2013/02821-1)

\section{Referências}

Baranauskas, M.C.C., 2009. Socially Aware Computing. In: Proc. of VI International Conference on Engineering and Computer Education (ICECE 2009), pp. 1-5.

Baranauskas, M.C.C.; Martins, M.C.; Valente, J.A. (2013). Codesign de Redes Digitais Tecnologia e Educação a Serviço da Inclusão. 1ed.: Editora Grupo A - Penso.

Baranauskas, M.C.C., Schimiguel, J., Simoni, C.A.C., Medeiros, C.M.B. (2005). Guiding the Process of Requirements Elicitation with a Semiotic Approach. In: Proc. of 11th International Conference on Human-Computer Interaction, pp. 100-111.

Bradley, M.M.; Lang, P.J. (1994) Measuring emotion: the Self-Assessment Manikin and the Semantic Differential. J Behav Ther Exp Psychiatry. 1994 Mar;25(1):49-59.

Buchdid, S.B., Baranauskas, M.C.C., (2012) IHC em contexto: o que as palavras revelam sobre ela, In: XI Simpósio Brasileiro de Fatores Humanos em Sistemas Computacionais (IHC'12), pp. 199-208.

Friedman, B., Kahn, P.H., Borning, A., (2006) Value sensitive design and information systems, In: Human-Computer Interaction and Management Information Systems: Foundations, Armonk, pp. 348-372.

Guha, M.L.; Druin, A.; Fails, J.A. (2010) Investigating the Impact of Design Processes on Children. Proc. of Interaction Design and Children (IDC'10).

Hayashi, E. C. S.; Martins, M. C.; Baranauskas, M. C. C. (2012) Schools as organizations: A semiotic approach towards making sense of information technology. In: Proceedings of the 14th International Conference on Enterprise Information System (ICEIS 2012), pp. 15-24.

Hayashi, E.C.S.; Baranauskas, M. C. C. 'Affectibility' and Design Workshops: Taking actions towards more sensible design. Proc. of the 12th Brazilian Symposium on Human Factors in Computing Systems (to be published).

John, B.E., Kieras, D.E., (1996). Using GOMS for User Interface Design and Evaluation: Which Technique? ACM Transactions on Computer-Human Interaction, 3, 287-319. 
Hall, E.T. (1959) The Silent Language, Anchoor Books, New York.

Isbister, K.; Höök, K.; Sharp, M.; Laaksolahti, J. (2006) The Sensual Evaluation Instrument: Developing an Affective Evaluation Tool. In: Proc. of ACM Conference on Human Factors in Computing Systems (CHI'06), pp. 1163-1172.

LaTaille, Y.(1992) Desenvolvimento do juízo moral e afetividade na teoria de Jean Piaget. Piaget, Vygotsky, Wallon: Teorias Psicogenéticas em Discussão. São Paulo: Summus Editorial.

Liu, X. (2001) Employing MEASUR Methods for Business Process Reengineering in China. Ph.D. Thesis University of Twente, Enschede, the Netherlands.

Muller, M.J., Haslwanter, J.H., Dayton, T., (1997) Participatory practices in the software lifecycle. In: Handbook of Human-Computer Interaction 2, pp. 255-297.

Melo, A. M.; Baranauskas, M.C.C.; Soares, S.C.M. (2008) Design com Crianças: da prática a um modelo de processo. Revista Brasileira de Informática na Educação, vol. 16(1), pp.43-55.

Nielsen, J. (1994) Heuristic Evaluation. In: Nielsen, J.; Mack, R. Usability Inspection Methods, 25-62.

ONU. Declaração dos Direitos da Criança. Disponível em: http://198.106.103.111/cmdca/downloads/Declaracao_dos_Direitos_da_Crianca.pdf (último acesso em jul., 2013)

Ortony, A., Norman, D.A. \& Revelle, W. (2005) Affect and Proto-affect in effective functioning. Who Needs Emotions? The Brain Meets the Machine. New York: Oxford University Press, pp. 173-202.

Pereira, R., Buchdid S.B., Miranda, L.C. and Baranauskas, M.C.C. (2012a) Considering Values and Cultural Aspects in the Evaluation of Interactive Systems Prototypes. In: International Conference on Information Society (i-Society 2012). pp. 395-400.

Pereira, R., Buchdid S.B. and Baranauskas, M.C.C. (2012b). Keeping Values in Mind: Artifacts for a Value-Oriented and Culturally Informed Design. In: 14th International Conference on Enterprise Information Systems (ICEIS 2012). pp. 25-34.

Pereira, R., Baranauskas, M. C. C., \& da Silva, S. R. P. (2013). Social Software and Educational Technology: Informal, Formal and Technical Values. Educational Technology \& Society, 16 (1), 4-14.

Russell, A. J. (1989) Affect Grid: A Single-Item Scale of Pleasure and Arousal". Journal of Personality and Social Psychology. Vol. 57(3), pp. 493-502.

Schwartz, S. H. (2005). Basic human values: Their content and structure across countries. In: Values and Behaviors in Organizations, Vozes, Rio de Janeiro.

Thomas, N.; O'Kane, C. (1998) The ethics of participatory research with children. Children \& Society, vol. 12, n. 5, pp. 336-348. 